\title{
Naked mole rat cells display more efficient DNA excision repair and higher resistance to toxic impacts than mouse cells
}

\author{
Alexey Evdokimov ${ }^{1}$, Irina Petruseva ${ }^{1}$, Aleksei Popov ${ }^{1}$, Olga Koval ${ }^{1}$, and Olga Lavrik $^{1,2}$ \\ ${ }^{1}$ Institute of Chemical Biology and Fundamental Medicine, 630090, Novosibirsk, Russian Federation \\ ${ }^{2}$ Novosibirsk State University, 630090, Novosibirsk, Russian Federation
}

\begin{abstract}
Naked mole rat is the long-lived and tumor-resistant rodent. Naked mole rat possesses multiple adaptations that may contribute to longevity and cancer-resistance. Higher activity of DNA excision repair systems and their faster recovery after genotoxic impact as compare with Mus musculus directly demonstrated in our previous investigation contribute to longevity and cancer resistance of naked mole rat. In the present study the DNA-damage-induced apoptosis in naked mole rat fibroblasts was studied using conventional methods. The ability of naked mole rat cells to undergo regulated cell death in response to genotoxic stress is another group of cell defense mechanisms. Naked mole rat skin fibroblasts were demonstrated to be much more resistant towards proapoptotic reagents methyl methanesulfonate, 5-fluorouracil and etoposide as compared with fibroblasts of Mus musculus. Naked mole rat cells have demonstrated limited apoptotic response and seem to undergo also other-type regulated cell death under severe genotoxic stress.
\end{abstract}

\section{Introduction}

Naked mole rat (NMR, Heterochephalus glaber) is the extremely long-lived and tumorresistant small rodent. NMRs possess multiple adaptations that may contribute to longevity and cancer-resistance: stable genome and epigenome, stable transcriptome, elevated stability of proteins and elevated expression of several mRNA coding DNA repair proteins were described and [1-5]. Recently we have demonstrated that NMR cells display more efficient excision repair than mouse cells [6]. We compared base excision repair (BER) and nucleotide excision repair (NER) in cell extracts from NMR and mouse (Mus musculus, MMU) fibroblasts after UV exposure. The non-irradiated cells and cells cultivated 1-24h after UVC-irradiation investigated. Time course of transcript levels of the key BER and NER proteins for most of mRNAs no sharp fluctuations was detected for both cells types, especially for NMR. In mouse cells, levels of several mRNA demonstrated more significant inducibility and variability. BER system status was characterized via activities of uracilDNA glycosylase, AP- endonuclease 1, and DNA polymerase. NMR cell extracts were more efficient at removing uracil and at cleaving the AP-site than the mouse ones. The higher level of poly(ADP-ribose) synthesis as well as PARP1 covalent binding to DNA probes containing apurinic/apyrimidinic sites or photo-reactive groups illustrates more 
powerful DNA damage response in the NMR cells. The effective excision of bulky damage occurs after primary recognition and verification and pre-incision and incision complexes assembling. More than 25 protein factors and enzymes participate in the process: XPC, TFIIH, RFC complexes, XPA, RPA, XPF-ERCC1, XPG [7]. Thus, a comparative evaluation of the specific excision efficiency makes it possible to compare NER system activity in cell extracts samples. Measurement of specific excision reaction demonstrated that all NMR extracts were more efficient than mouse ones at bulky lesion elimination. Summarizing, our results suggest that NMR has more efficient NER and BER systems than the mouse, which may contribute to longevity and cancer resistance of this species.

The genome stability maintaining, cell resistance to cancer neoplasia and survival under damaging impacts is a function of the repair capacity and regulated cell death mechanisms. NMR cells were found to be more resistant than mouse cells to a variety of toxicants such as cadmium ions, MMS, paraquat, rotenone, heat, and low glucose media. Despite the upregulated expression of some BER and NER related genes [8,9], NMR fibroblasts were more sensitive to $\mathrm{H}_{2} \mathrm{O}_{2}$ and UV light [10]. Apoptosis is very important mechanism of RCD in multicellular organisms. The apoptosis program is essential for the development, removal of dangerous cells, as well as cellular homeostasis [11]. Defects in apoptosis regulation lead to a number of malignancies such as cancer and autoimmune diseases. Seluanov et al. reported that the spontaneous apoptosis level in NMR fibroblasts is low (no higher than $7 \%$ for skin fibroblasts) and is characterized by very specific regulation [12].

The aim of the present investigation was to study the resistance of NMR cells towards methyl methane sulfonate (MMS), 5-fluorouracil (5FU) and etoposide (Eto), which are widely used as proapoptotic reagents, to uncover the mechanisms of regulated cell death in naked mole rat.

\section{Methods}

\section{Cell culture}

The establishment of cell culture derived from NMR fibroblasts was described earlier [13]. NMR skin fibroblasts NSF8 cells were kindly provided by Dr V. Gorbunova (University of Rochester, Department of Biology) NMR cells cultured in MEM supplemented with 15\% FBS (Gibco), 10\% AmnioMAX II Complete Medium (Gibco), 5 ng/ml bFGF, $10^{5} \mathrm{U} / \mathrm{L}$ penicillin, $100 \mathrm{mg} / \mathrm{L}$ streptomycin, and $2.5 \mathrm{mg} / \mathrm{L}$ amphotericin $\beta$ at $32^{\circ} \mathrm{C}$ in the presence of $5 \% \mathrm{CO}_{2}$. The cell line was deposited in the cell bank of the Institute of Molecular and Cellular Biology, SB RAS ("The General Collection of Cell Cultures", No. 0310-20160002).

The 3 T3 mouse cell line was kindly provided by Dr. V. Fishman (Sector of Genomic Mechanisms of Ontogenesis, Institute of Cytology and Genetics, Russia). Cells were cultured in MEM supplemented with $15 \%$ FBS (Gibco), $10^{5} \mathrm{U} / \mathrm{L}$ penicillin, $100 \mathrm{mg} / \mathrm{L}$ streptomycin, and $2.5 \mathrm{mg} / \mathrm{L}$ amphotericin $\beta$ at $37^{\circ} \mathrm{C}$ in the presence of $5 \% \mathrm{CO}_{2}$.

All the subsequent experiments were carried out with dividing cells in the exponential growth phase.

\section{Cell viability assay}

Cells were seeded in 96-well plates at a concentration of $3 \times 10^{3}$ cells/well in $200 \mu 1$ culture medium and cultured as described above for 24 (NSF8 cells: NMR fibroblasts) or 12 (3T3 cells: Mouse fibroblasts (MMU)) h. Afterwards the compounds dissolved in DMSO were added, and the cells were incubated for various time intervals. The EZ4U test (Biomedica) 
was carried out according to the manufacturer's instructions. Briefly, $20 \mu \mathrm{l}$ of EZ4U labeling mixture was added, and the cells were incubated for 2-3 h. Absorbance of the samples was measured at $492 \mathrm{~nm}$ using a microplate reader. All experiments were repeated in the triplicate.

\section{Caspase activity detection}

Cells were seeded in 96-well plates at a concentration of $3 \times 10^{3}$ cells/well in $200 \mu$ culture medium and cultured as described above to reach $60 \%$ confluence. Caspase activity was measured using the Caspase-Glo 3/7 assay kit according to the manufacturer's instructions (Promega, Madison, WI). Briefly, $200 \mu \mathrm{l}$ of Caspase-Glo 3/7 reagent was added to each well; the plate was shaken gently for $30 \mathrm{~s}$ and incubated at room temperature for $2 \mathrm{~h}$. Luminescence of the samples was measured using an Infinite M200 plate reader (Tecan, Research Triangle Park, USA).

\section{Apoptosis detection}

NMR (NSF8 cells) and MMU (3T3 cells) cells were seeded in 6-well plates and cultured as described above. When the cell cultures reached $80 \%$ confluence, the cells were treated with the DNA-damaging compounds for 16 (5-fluorouracil) or 20 (etoposide and methyl methanesulfonate) h. Before trypsinization, detached cells were collected. Adherent cells were rinsed with PBS and detached from the plate with trypsin. Soybean trypsin inhibitor (Sigma) was added up to $10 \mu \mathrm{g} / \mathrm{ml}$ concentration to inhibit trypsin-initiated proteolysis. The detached and trypsinized cells were combined and pelleted by centrifugation ( 5 min at 500 g). FITC-Annexin V Apoptosis detection kit and FACSCanto II (BD Biosciences, San Jose, USA) flow cytometer were used to detect cell death. The analysis was performed on a FACSCantoII flow cytometer (Becton Dickinson) using the FACSDiva Software (BD Biosciences). Cells were initially gated based on forward scatter vs. side scatter to exclude small debris. Ten thousand events from each population were collected.

\section{Results and Discussion}

Three DNA damaging agents having different mechanisms of action, methyl methanesulfonate (MMS), 5-fluorouracil and etoposide were used to compare the sensitivity of skin fibroblasts from HLG and MMU. The sensitivity of NMR and mouse fibroblasts to stress induced by the selected reagents was evaluated using metabolic assays. To have similar conditions for this comparative analysis the cells from both NMR vs. MMU were taken in the exponential growth phase. The skin fibroblasts from NMR vs. MMU, e.g. NSF8 and 3T3 cells, respectively, were treated in the time- and dose- dependent manner with MMS, 5-fluorouracil and etoposide. The significant loss of cell viability of NMR fibroblasts was observed only starting from the concentration of $100 \mu \mathrm{M}$ (Fig. 1). Indeed, the treatment within conventional concentrations used in the studies of DNA damage-induced apoptosis (range between 10 and $70 \mu \mathrm{M}$ ) for all three DNA damaging reagents led to the significant cell viability loss of MMU cells. At $20 \mathrm{~h}$ time point after treatment with MMS, 5-fluorouracil and etoposide the metabolic activity for MMU cells was 50, 40 and 30\% while for NMR cells it was 70, 90 and $80 \%$ respectively. It can be concluded that MMU fibroblasts are more sensitive to DNA damage-induced cell viability loss compared to the fibroblasts from NMR. 

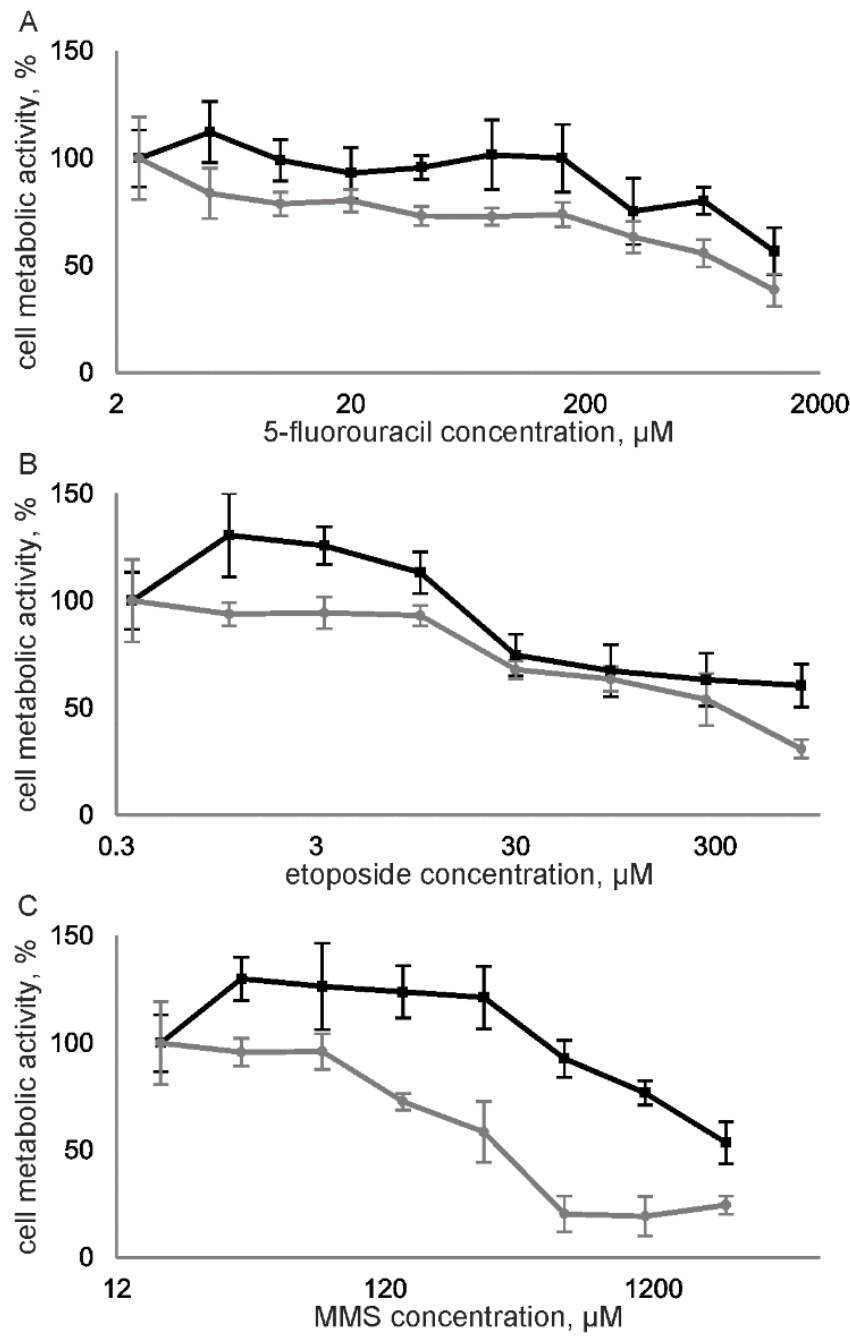

Fig. 1. Metabolic activity of NMR (black lines) and MMU (grey lines) cells after treatment with 5fluorouracil (A), etoposide (B) and MMS (C).

The method of flow cytometry analysis was used to investigate the cell death of the NMR fibroblasts. Flow cytometry assay with FITC-Annexin V and propidium iodide staining was used to perform the analysis of cell populations exposed to genotoxic stress conditions. A range of reagent concentrations and the exposure time were chosen in accordance with the data obtained by evaluating the metabolic activity and post-induction time dependence of caspase activation. (See Materials and Methods for more details). For each concentration of each genotoxic agent the percentages of viable cells, early apoptotic cells, and cells that have been late apoptotic stage or have undergone necrotic changes were calculated. Changes in cell viability were observed within the selected range of stress conditions (7.5-120 $\mu \mathrm{M}$ etoposide, 35-560 $\mu \mathrm{M}$ MMS, and 10-160 $\mu \mathrm{M}$ 5-fluorouracil) with eexposure time to etoposide for $20 \mathrm{~h}$; to MMS and 5-fluorouracil for $16 \mathrm{~h}$. The relative number of viable, apoptotic and necrotic cells was then measured (Fig. 2). 

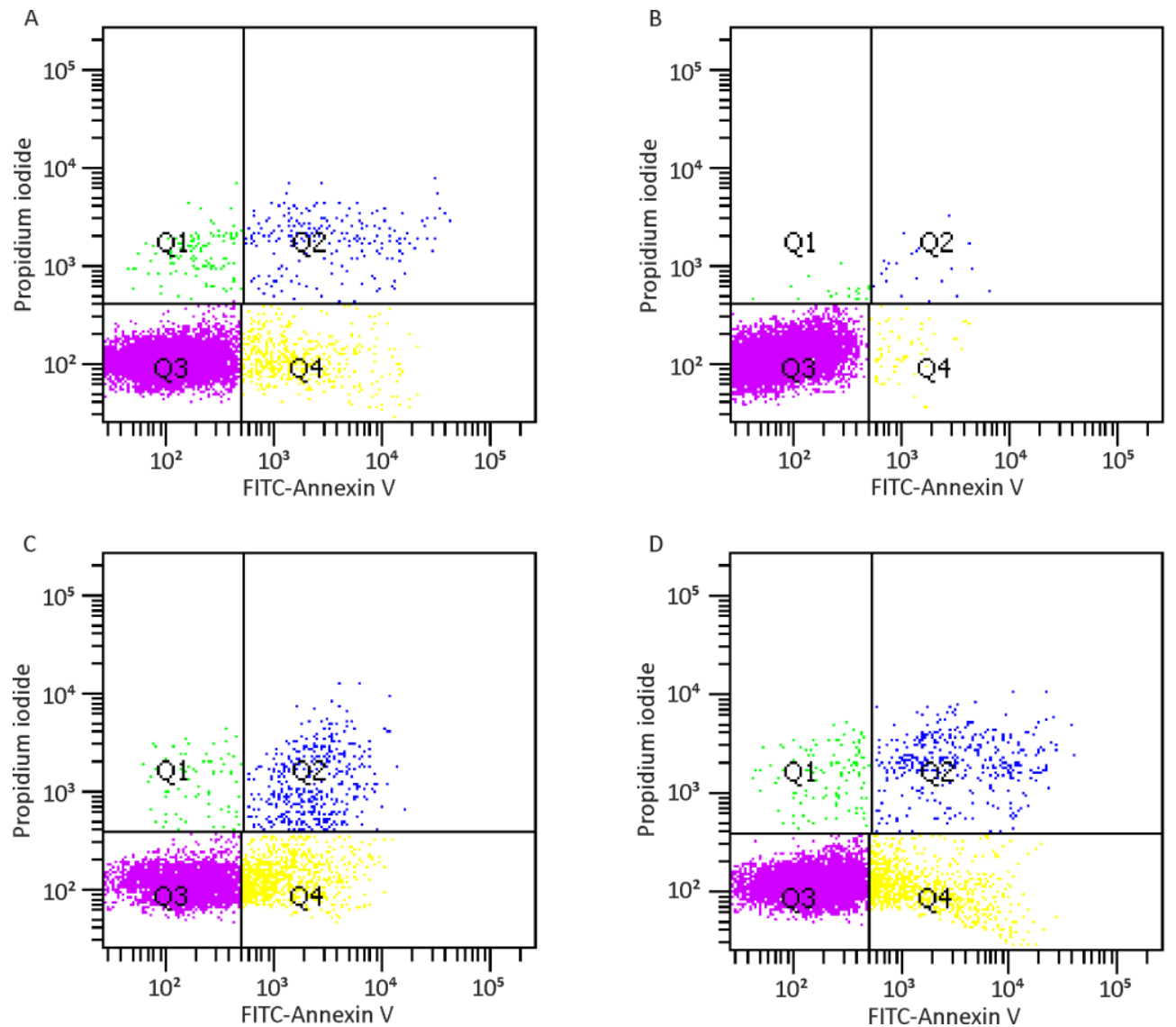

Fig. 2. FACS analysis of native (panels A, B) cell and cells upon treatment with $60 \mu \mathrm{M}$ etoposide (C, D) of MMU (A, C) and NMR (B, D).

Unlike mouse cells, naked mole rat cells are especially resistant to some proapoptotic reagents (e.g., 5-fluorouracil as it was demonstrated in this study). The data obtained using evaluation of metabolic activity, activity of caspases and flow cytometry assay, give grounds for assuming lower efficiency of apoptosis activation in NMR than in mice. when exposed to high-dose toxic agents, the naked mole rat cells demonstrate tendency to rather necrotic than apoptotic death.

\section{Conclusions}

The comparative studies showed that cells of long-lived and cancer-prone NMR are highly resistant to all types of DNA-damaging agents used. It is fair to assume that the differences in responses of NMR and mouse cells to the exposure of DNA damaging agents correspond to previously higher efficiency of DNA repair systems in NMR cells in comparison to mouse ones [6]. The limited activation of proapoptotic cascade and low intensity of apoptotic response may be related with a special type of regulation in NMR [12] and stability of the expression level under the toxic impacts [6]. allow to suppose a significant contribution of other RCD mechanisms to the maintenance of NMR stable functioning. 


\section{Acknowledgments}

This study was supported by the Russian Science Foundation (project no. 19-74-10056).

We would like to express our thanks to Prof. V. Gorbunova (University of Rochester, NY, USA) for kindly provided NMR cell culture NSF8, Dr. V. Fishman (Sector of Genomic Mechanisms of Ontogenesis, Institute of Cytology and Genetics, Novosibirsk, Russia) for kindly provided 3T3 cells. The authors also thank Dr. V. Trifonov, Dr. S. Romanenko and Prof. A. Graphodatsky (Institute of Molecular and Cellular Biology, Novosibirsk, Russia) for kind cooperation, Prof. I. Lavrik for careful reading of the manuscript and valuable comments.

\section{References}

1. E. B. Kim, X. Fang, A. A. Fushan, Z. Huang, A. V. Lobanov, L. Han, S. M. Marino, X. Sun, A. A. Turanov, P. Yang, S. H. Yim, X. Zhao, M. V. Kasaikina, N. Stoletzki, C. Peng, P. Polak, Z. Xiong, A. Kiezun, Y. Zhu, Y. Chen, G. V. Kryukov, Q. Zhang, L. Peshkin, L. Yang, R. T. Bronson, R. Buffenstein, B. Wang, C. Han, Q. Li, L. Chen, W. Zhao, S. R. Sunyaev, T. J. Park, G. Zhang, J. Wang, and V. N. Gladyshev, Nature 479, $223(2011)$

2. X. Fang, I. Seim, Z. Huang, M. V Gerashchenko, Z. Xiong, A. A. Turanov, Y. Zhu, A.V. Lobanov, D. Fan, S. H. Yim, X. Yao, S. Ma, L. Yang, S.-G. Lee, E. B. Kim, R. T. Bronson, R. Šumbera, R. Buffenstein, X. Zhou, A. Krogh, T. J. Park, G. Zhang, J. Wang, V. N. Gladyshev, Cell Rep., 8, 1354 (2014)

3. I. O. Petruseva, A. N. Evdokimov, O. I. Lavrik, Acta Naturae, 9, 31 (2017)

4. N. Labinskyy, A. Csiszar, Z. Orosz, K. Smith, A. Rivera, R. Buffenstein, Z. Ungvari, Am. J. Physiol. - Hear. Circ. Physiol., 291, H2698 (2006)

5. L. Tan, Z. Ke, G. Tombline, N. Macoretta, K. Hayes, X. Tian, R. Lv, J. Ablaeva, M. Gilbert, N.V Bhanu, Z. Yuan, B.A. Garcia, Y.G. Shi, Y. Shi, A.Seluanov, V. Gorbunova, Stem Cell Reports, 9, 1721 (2017)

6. A. Evdokimov, M. Kutuzov, I. Petruseva, N. Lukjanchikova, E. Kashina, E. Kolova, T. Zemerova, S. Romanenko, P. Perelman, D. Prokopov, A. Seluanov, V. Gorbunova, A. Graphodatsky, V. Trifonov, S. Khodyreva, and O. Lavrik, Aging (Albany. NY), 10, 1454 (2018)

7. C. Li, F. M. Golebiowski, Y. Onishi, N. L. Samara, W. Yang, 59, 1025 (2015)

8. S. Ma, A. Upneja, A. Galecki, Y.-M. Tsai, C. F. Burant, S. Raskind, Q. Zhang, Z. D. Zhang, A. Seluanov, V. Gorbunova, C. B. Clish, R. A. Miller, V. N. Gladyshev, Elife, 5, e19130 (2016)

9. L. Macrae, M. M. Croken, R. B. Calder, A. Aliper, R. R. White, A. Zhavoronkov, V. N. Gladyshev, A. Seluanov, V. Gorbunova, Z. D. Zhang, J. Vijg, 7, 1171 (2015)

10. A. B. Salmon, A. A. Sadighi Akha, R. Buffenstein, R. A. Miller, Journals Gerontol. Ser. A Biol. Sci. Med. Sci., 63, 232 (2008)

11. P. H. Krammer, R. Arnold, I. N. Lavrik, Nat. Rev. Immunol., 7, 532 (2007)

12. A. Seluanov, C. Hine, J. Azpurua, M. Feigenson, M. Bozzella, Z. Mao, K. C. Catania, V. Gorbunova, Proc. Natl. Acad. Sci. U. S. A., 106, 19352 (2009)

13. X. Tian, J. Azpurua, Z. Ke, A. Augereau, Z.D. Zhang, J. Vijg, V.N. Gladyshev, V. Gorbunova, A. Seluanov, Proc. Natl. Acad. Sci. U. S. A., 112, 1053 (2015) 